\title{
Outcomes related to respiratory syncytial virus with an abbreviated palivizumab regimen in children with congenital heart disease: a descriptive analysis
}

\author{
Jennifer Claydon MSc, Constantin R. Popescu MD MSc, Lana Shaiba MBBS MS, \\ Cheryl Christopherson BScN, Derek Human MB BCh, Richard Taylor MBBS, Alfonso Solimano MD, \\ Pascal M. Lavoie MD PhD
}

\section{Abstract}

Background: It has been hypothesized that 4 doses of palivizumab, a neutralizing monoclonal antibody against respiratory syncytial virus (RSV), administered during a fixed-date RSV season may reduce hospital admissions comparably to the standard 5-dose schedule. We report outcomes in children with congenital heart disease approved to receive this 4-dose palivizumab schedule in British Columbia.

Methods: We performed a population-based descriptive cohort analysis of all 406 approved palivizumab courses over 4 seasons (2012/13 to 2015/16) in 325 children with hemodynamically significant congenital heart disease enrolled in the British Columbia RSV Immunoprophylaxis Program. The primary outcome was in-season hospital admission for potential RSV-related lower respiratory tract infection (LRTI). Secondary outcomes include timing of admission in relation to dosing. Analysis was by intention-to-treat.

Results: Of the 406 approved palivizumab courses, 391 were administered. In 33 cases (8.4\%), an additional dose was given immediately after cardiac bypass surgery. There were 17 RSV-confirmed hospital admissions (median age of children 5.9 mo [interquartile range 4-10 mo]) and 8 admissions in which the child was not tested for RSV, for a maximum of 25 potential RSV-related admissions (6.2 per 100 approvals [95\% confidence interval 4.0-9.0]). Twenty-four (96\%) of the 25 admissions occurred within the 4-dose palivizumab dosing period, and the remaining admission occurred 52 days after the fourth dose. Sixty-four (72\%) of 89 admissions were RSV-negative; the baseline clinical characteristics of these children were not different from those of children with RSV-confirmed admissions.

Interpretation: In infants with hemodynamically significant congenital heart disease, a 4-dose fixed-date palivizumab schedule over a 6-month season provided seasonal protection comparable to that in a clinical trial involving a standard 5-dose schedule. Because RSV was responsible for only $19 \%$ of admissions for LRTI in our cohort, it is critical to continue to emphasize other preventive measures, including family education toward proper hand hygiene, breast-feeding and limiting infectious exposures in children at high risk.

espiratory syncytial virus (RSV) is a leading cause of lower respiratory tract infection (LRTI) in young children. ${ }^{1}$ Infants born prematurely and those with chronic lung or congenital heart diseases are at higher risk. ${ }^{2,3}$ Most hospital admissions occur during the winter, the socalled RSV season, which, in most jurisdictions, lasts 6 months. ${ }^{4}$ There is no vaccine against RSV. However, in a randomized controlled trial, 5 seasonal doses of palivizumab, a neutralizing monoclonal antibody against RSV, reduced rates of hospital admission in children less than 2 years of age with congenital heart disease, from 9.7\% (95\% confidence interval [CI] $7.7 \%-12 \%)$ in the placebo group to $5.3 \%(95 \% \mathrm{CI}$ $3.8 \%-7.3 \%$ ) in the palivizumab group. ${ }^{5}$ Thus, contrary to conventional vaccines, which are supposed to induce nearly com- plete protection in a majority of the population, palivizumab is a passive monoclonal antibody that provides only partial (about $50 \%$ ) protection as long as sufficiently elevated serum drug levels persist during the period of high viral exposure. ${ }^{6}$

A key question, then, is how many doses are necessary to achieve optimal protection over the typical 6-month RSV

Competing interests: None declared.

This article has been peer reviewed.

Correspondence to: Pascal Lavoie, plavoie@cw.bc.ca

CMAJ Open 2019. DOI:10.9778/cmajo.20180167 
season? Several authors have suggested that a 4-dose schedule should perform nearly as well as the established 5-dose schedule in most settings while reducing unnecessary clinic visits for families, drug injections for children and costs to the health care system. ${ }^{4,7-10}$ To our knowledge, British Columbia is the only jurisdiction that uses abbreviated 4-dose, fixed-date schedules. ${ }^{3,10}$ Our group recently reported outcomes for infants in the BC RSV Immunoprophylaxis Program overall. ${ }^{11}$ However, children with congenital heart disease constitute a distinct subgroup of patients who tend to have more severe RSV infections, with hospital admission rates as high as $36 \%$ in the absence of palivizumab therapy. ${ }^{12} \mathrm{We}$ report rates of hospital admission in children with congenital heart disease who were approved to receive an abbreviated 4-dose palivizumab schedule in BC.

\section{Methods}

\section{Study design and setting}

This was a descriptive population-based cohort study of all children with congenital heart disease in the BC RSV Immunoprophylaxis Program over 4 consecutive seasons (2012-2016); this defined our sample size. The program centrally manages all palivizumab administration provincially. Children less than 12 months of age with hemodynamically significant congenital heart disease are universally approved to receive 4 doses. Children aged 12-24 months with congenital heart disease may also receive 4 palivizumab doses after clinical review by an expert panel, based on best available evidence.

\section{Exposure}

Within the RSV Immunoprophylaxis Program, palivizumab is administered according to prespecified fixed season dates starting on the third Monday of November until Mar. 31. This period was defined based on a review of 16 seasons (1994-2011) of provincial RSV hospital admission data. ${ }^{13}$ After reviewing the pharmacokinetics of palivizumab, the RSV Immunoprophylaxis Program concluded that 4 doses would confer sufficient protective serum drug levels over a typical 6-month RSV season. ${ }^{10}$ Both the American Academy of Pediatrics and the Canadian Paediatric Society recommend against palivizumab administration in infants who are still in hospital, instead favouring protecting them by means of infection-control principles. ${ }^{2,3}$ Accordingly, in BC, palivizumab is administered only to ambulatory children or just before hospital discharge.

Palivizumab is administered by program clinic nurses distributed across the province (87\% of cases) or in family doctors' offices (13\% of cases). Children receive up to 4 doses of $15 \mathrm{mg} / \mathrm{kg}$ administered intramuscularly; the second dose is given 21 days (maximum $28 \mathrm{~d}$ ) after the first dose, and subsequent doses are given 28 days (maximum 35 d) apart. If an infant undergoes cardiopulmonary bypass during the season, 1 additional dose is administered (immediately after the procedure). No doses are administered after Mar. 31 of a given season.

\section{Outcomes and follow-up period}

The main outcome was potential RSV-related hospital admission, which included RSV-confirmed or RSV-undetermined LRTI, as defined below. Follow-up occurred between Nov. 1 and Apr. 30 for each season.

\section{Data collection and definitions}

Hospital admissions were centrally reported by clinic nurses to the RSV Immunoprophylaxis Program Provincial Clinic Coordinator. In addition, we extracted admissions for LRTI from the Canadian Institute for Health Information Discharge Abstract Database from health authorities in BC using 7 International Statistical Classification of Diseases and Related Health Problems, 10 Revision diagnostic codes: B97.4 - Respiratory syncytial virus as the cause of diseases classified to other chapters, J12.1 - Respiratory syncytial virus pneumonia, J12.9 - Viral pneumonia, unspecified, J20.5 - Acute bronchitis due to respiratory syncytial virus, J20.9 - Acute bronchitis, unspecified, J21.0 - Acute bronchiolitis due to respiratory syncytial virus and J21.9 - Acute bronchiolitis, unspecified.

The diagnosis of LRTI was confirmed by a review of the medical chart, including results of viral testing (nasopharyngeal swabs) whenever available (L.S., C.R.P., J.C., C.C.). Hospital admissions of children with a positive result of testing for RSV were considered RSV-confirmed, and those of children not tested for RSV were considered RSV-undetermined. We included both categories in conservative estimates of RSVrelated admissions. Admissions of children with a negative result of RSV testing were considered RSV-negative. The data were compiled electronically in a spreadsheet, according to whether the admission occurred before receipt of the first dose of palivizumab (before or, in the case of "late" referrals, after the season start), within the dosing period (including up to $35 \mathrm{~d}$ after the fourth dose) or more than 35 days after receipt of the fourth palivizumab dose. Mortality is reported as of the end of the 2016 follow-up period for all children. All data were manually reviewed for accuracy by 2 investigators (J.C., P.M.L).

In addition, we analyzed the following baseline demographic data: type of congenital heart disease, whether a surgical correction was performed (including date and whether cardiopulmonary bypass was required) and receipt of palivizumab doses (with dates). Congenital heart diseases were categorized by a cardiologist (D.H.) as cyanotic lesion, left-to-right shunt lesion, left ventricular obstruction (including coarctation of the aorta), combined lesions, cardiomyopathy or unspecified heart defect. Any missing data were treated as such and reported in the tables.

\section{Statistical analysis}

We analyzed the data per child per RSV season, except for data related to children's demographic characteristics at baseline and between RSV-confirmed and RSV-negative hospital admissions. This means that, if a child received palivizumab for more than $1 \mathrm{RSV}$ season, we counted each season as a separate event (referred to as "approvals"). We analyzed the results descriptively using $95 \%$ CIs calculated by means of the 
binomial exact method (www.sample-size.net/confidence -interval-proportion/), standard deviations or interquartile range (depending on expected data distribution). Analysis was by intention-to-treat.

\section{Ethics approval}

This study was approved by the Children's \& Women's Health Centre of British Columbia Research Ethics Board.

\section{Results}

\section{Study population}

The clinical characteristics of the 325 children included in the study are presented in Table 1 . Most were born at term (236/318 [74.2\%] for whom gestational age data were available) and were younger than 6 months at the start of the RSV season (242 [74.5\%]). Nearly half (151 [46.5\%]) had cyanotic heart defects, 129 (39.7\%) had hemodynamically significant left-right shunt, 18 (5.5\%) had left ventricular outflow tract obstruction, 15 (4.6\%) had cardiomyopathy, 9 (2.8\%) had a combination of complex anomalies, and 3 (0.9\%) had unspecified heart defects.

\section{Palivizumab administration}

Among the 325 children, 406 palivizumab courses were approved. Most of the courses (288 [70.9\%]) were approved during the child's first season, 100 courses $(24.6 \%)$ were approved in the second season, and 18 courses (4.4\%) were approved in the third season. Seventy-two children $(22.2 \%)$ received palivizumab for more than 1 season.

Of the 406 approved courses of palivizumab, 391 were administered (Figure 1). Twelve were never administered because of parent/legal guardian refusal (10 cases) or a prolonged hospital stay during the season (2 cases). In 3 cases, the number of palivizumab doses could not be determined.

Of the 391 palivizumab courses administered, $351(89.8 \%)$ included up to 4 doses, and 38 (9.7\%) included an additional

\section{Table 1: Clinical characteristics of 325 children with congenital heart disease enrolled in the British Columbia RSV Immunoprophylaxis Program}

\begin{tabular}{|lc|}
\hline Characteristic & $\%(95 \% \mathrm{Cl})^{*}$ \\
\hline Gestational age, wk, mean $\pm \mathrm{SD}(n=318)$ & $36.9 \pm 6.2$ \\
\hline Birth weight, g, mean $\pm \mathrm{SD}(n=319)$ & $2870 \pm 871$ \\
\hline Male sex $(n=324)$ & $52(46-57)$ \\
\hline $\begin{array}{l}\text { Age at start of first season, mo, median } \\
(\mathrm{IQR})\end{array}$ & $2.2(0-6.0)$ \\
\hline Surgical correction† $(n=315)$ & $51(45-57)$ \\
\hline Required bypass procedure $(n=288)$ & $34(29-40)$ \\
\hline Died & $4.0(2.1-6.7)$ \\
\hline $\begin{array}{l}\text { Note: } \mathrm{Cl}=\text { confidence interval, IQR }=\text { interquartile range, RSV }=\text { respiratory } \\
\text { syncytial virus, SD }=\text { standard deviation. } \\
\text { *Except where noted otherwise. } \\
\text { †For the congenital heart defect during the follow-up period. }\end{array}$ \\
\hline
\end{tabular}

fifth dose received, immediately after cardiac bypass surgery in 33 cases $(8.4 \%)$ and at the physician's discretion in 5 cases $(1.3 \%)$. Two courses $(0.5 \%)$ included 6 doses, at the physician's discretion. Another 30 courses (7.7\%) were not completed, 7 owing to noncompliance during the season, 3 as a result of hospital admission, 4 owing to the child's death, 2 at the treating physician's discretion and 14 for unspecified reasons.

\section{Outcomes}

There was 1 hospital admission among 64 approvals and more than 1 admission among 17 approvals, for a total of 89 admissions $(21.9 \%)$ among the 406 approvals over 4 seasons (Figure 2).

In 17 admissions, the child had a positive result of RSV testing, for a rate of RSV-confirmed admissions of 4.2 per 100 season-approvals (95\% CI $2.5 \%-6.6 \%$ ). In 8 cases, the child was not tested for RSV, for a maximum rate of potential RSV-related admissions of 6.2 per 100 approvals (95\% CI $4.0 \%-9.0 \%)$. Sixty-four children $(64 / 406=15.8 \%)$ had positive results of testing for non-RSV pathogens.

Of the 25 children with RSV-confirmed or RSVundetermined admission, 20 were in their first RSV season and 5 were in their second season. Twenty-three (92\%) of the 25 children were aged 12 months or less, and the other 2 children were 21 and 22 months, respectively. Of the 2 latter children, 1 had a corrected transposition of the great arteries with atrioventricular discordance and significant residual systemic (left) outflow tract obstruction, and the other had Down syndrome.

Among the 17 RSV-confirmed admissions, 2 occurred after the start of the season, on Nov. 19 and Nov. 27, but before an RSV Immunoprophylaxis Program application had been received. Fourteen admissions occurred within the dosing period. In the remaining admission, the child presented 52 days after receipt of the fourth dose. Among the $8 \mathrm{RSV}$ undetermined admissions, 1 occurred before a program application had been received, on Nov. 30, and 7 occurred within the dosing period ( 2 of which were nonadherent with the dosing schedule), including 1 that occurred at the latest 29 days after receipt of the fourth palivizumab dose.

The clinical characteristics of the children admitted for RSV-confirmed LRTI were similar to those admitted for RSV-negative LRTI (Table 2). No deaths were attributable to LRTI in this cohort. None of the 10 children whose parent/legal guardian refused palivizumab or of the 3 children whose number of doses could not be determined had a hospital admission related to an LRTI.

\section{Interpretation}

We report RSV-related hospital admissions among children with congenital heart disease who were approved to receive BC's 4-dose fixed-season abbreviated palivizumab schedule. We found that, in a geographical area where the RSV season consistently spans from early November to late April, all but 1 RSV-related hospital admission (24/25 [96\%]) occurred 


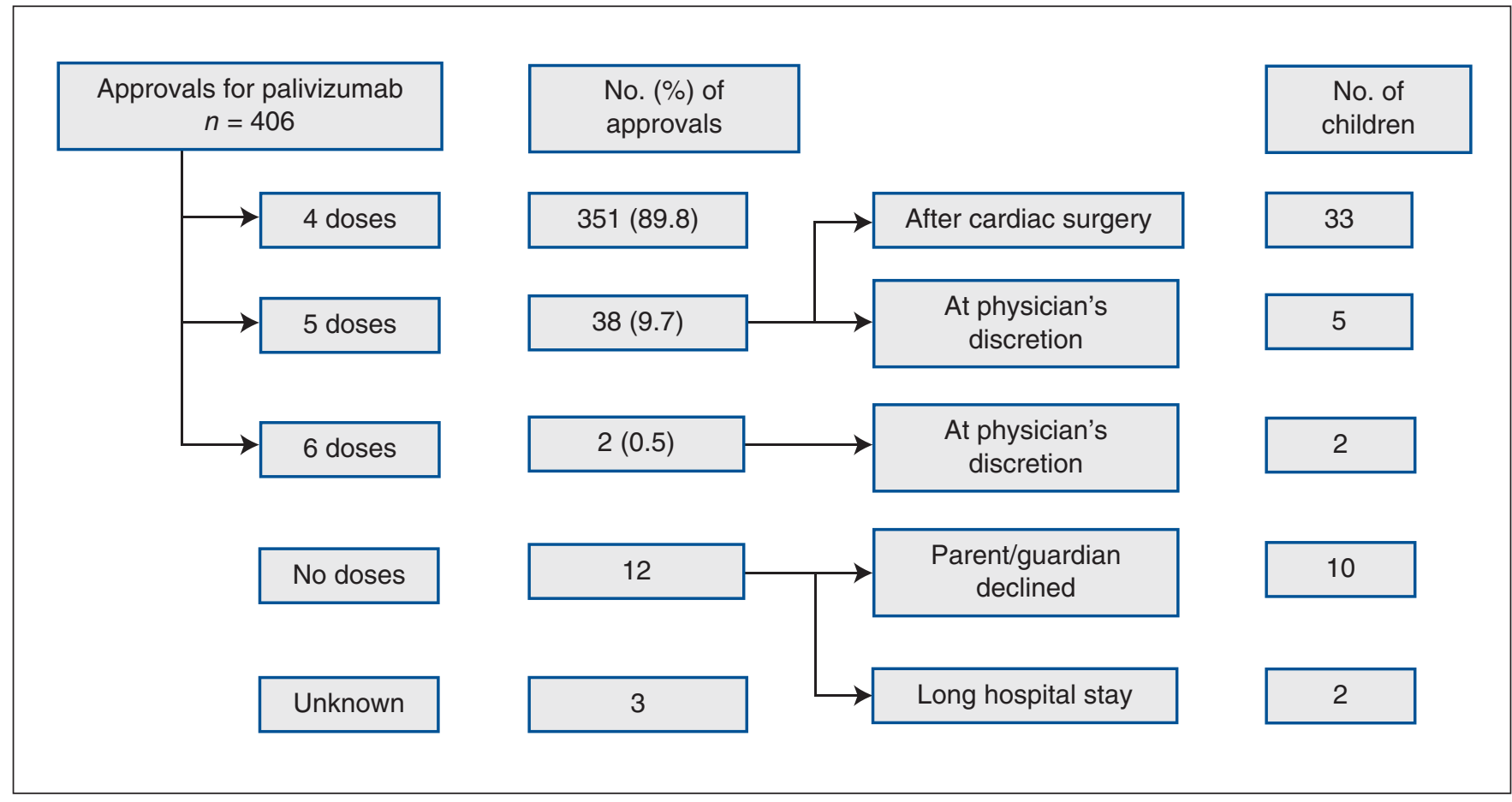

Figure 1: Intention-to-treat distribution of palivizumab doses administered to young children with congenital heart disease in British Columbia, November 2012 to April 2016.

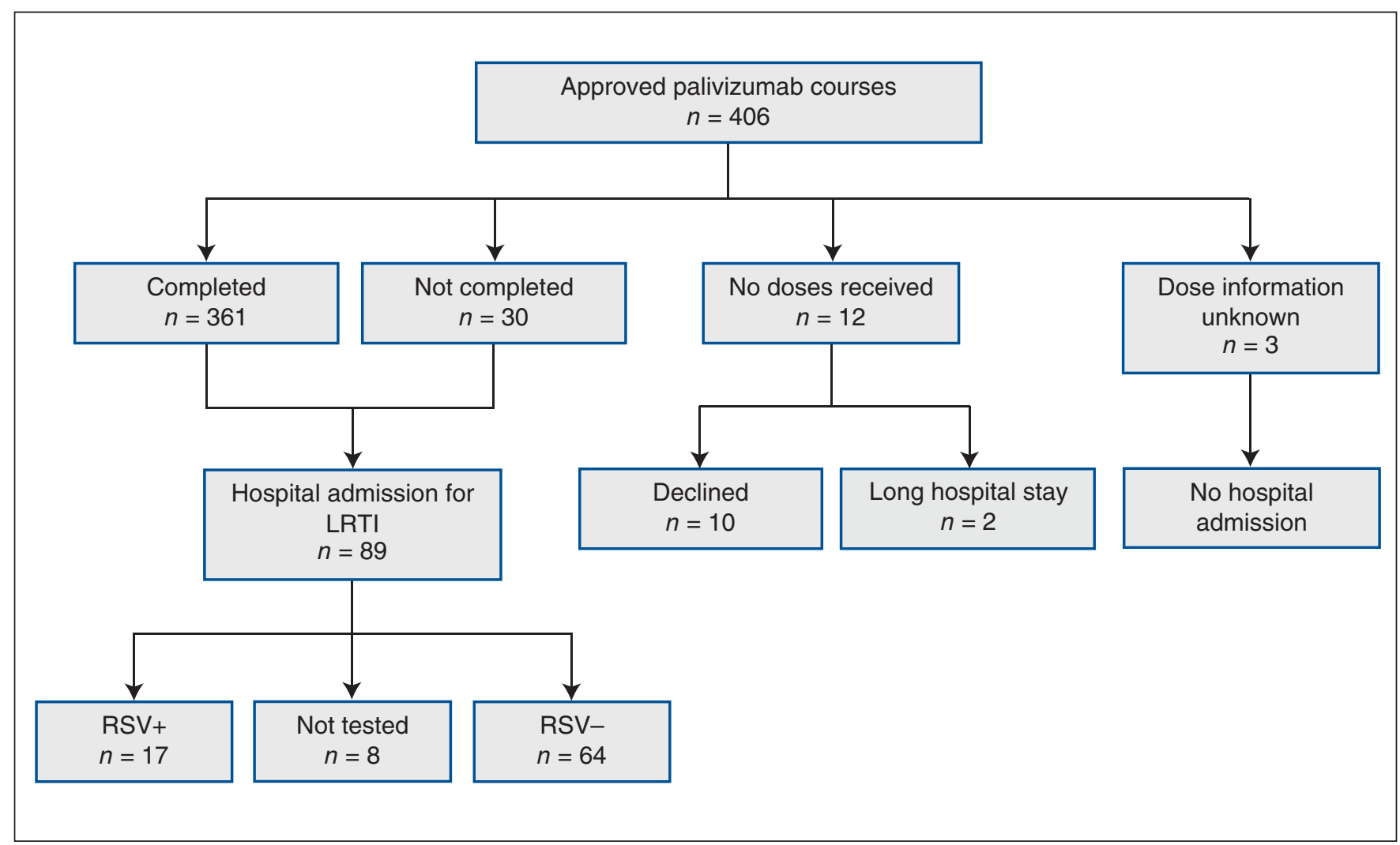

Figure 2: Hospital admissions for lower respiratory tract infection (LRTI), November 2012 to April 2016. Data are reported as number of children-approvals (i.e., approvals for each season in children who received palivizumab for more than 1 season are counted separately). Note: $\mathrm{RSV}=$ respiratory syncytial virus. 


\begin{tabular}{|c|c|c|}
\hline \multicolumn{3}{|c|}{$\begin{array}{l}\text { Table 2: Clinical characteristics of infants with RSV- } \\
\text { confirmed lower respiratory tract infection versus those } \\
\text { whose admissions for lower respiratory tract infection were } \\
\text { RSV-negative }\end{array}$} \\
\hline Characteristic & $\begin{array}{l}\text { RSV- } \\
\text { confirmed } \\
n=17\end{array}$ & $\begin{array}{c}\text { RSV-negative } \\
n=40\end{array}$ \\
\hline $\begin{array}{l}\text { Gestational age, wk, mean } \\
\pm \text { SD }\end{array}$ & $37.7 \pm 2.5$ & $37.8 \pm 2.3$ \\
\hline Birth weight, g, mean \pm SD & $2939 \pm 748$ & $2778 \pm 651$ \\
\hline Male sex, \% (95\% Cl) & 47 (23-72) & $50(34-66)$ \\
\hline $\begin{array}{l}\text { Age at first in-season } \\
\text { hospital admission, mo, } \\
\text { median (IQR) }\end{array}$ & $5(4-10)$ & $6(4-13)$ \\
\hline \multicolumn{3}{|l|}{$\begin{array}{l}\text { Type of congenital heart } \\
\text { disease, }{ }^{*} \%(95 \% \mathrm{Cl})\end{array}$} \\
\hline Cyanotic & $47(23-72)$ & $50(34-66)$ \\
\hline Left-right shunt & $35(14-62)$ & 33 (19-49) \\
\hline Obstructive lesion & $12(1.5-36)$ & $5(0.6-17)$ \\
\hline Cardiomyopathy & $6(0.0-29)$ & $2(0.0-13)$ \\
\hline Combination & 0 & $10(2.8-24)$ \\
\hline $\begin{array}{l}\text { Surgical correction, \% } \\
(95 \% \mathrm{Cl})\end{array}$ & $47(23-72)$ & $48(32-64) \dagger$ \\
\hline $\begin{array}{l}\text { Required bypass procedure, } \\
\%(95 \% \mathrm{Cl})\end{array}$ & $25(7.3-52) \ddagger$ & $40(24-58) \S$ \\
\hline Died, \% (95\% Cl) & $12(1.5-36)$ & $7(1.5-20)$ \\
\hline \multicolumn{3}{|c|}{ 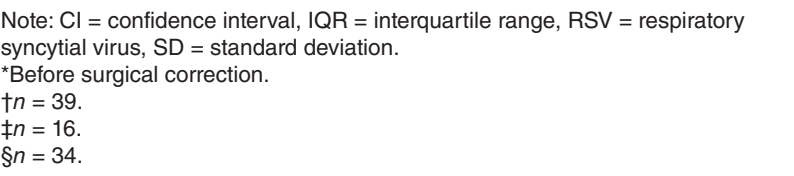 } \\
\hline
\end{tabular}

before administration of the fourth dose, and not as a result of starting immunoprophylaxis late in the season. Therefore, these cases were also not the result of a schedule failure. This result is consistent with the fact that the vast majority of RSV infections in North America and Europe occur in December and January, months during which coverage with palivizumab is expected to be no different between 4- and 5-dose schedules, and with the fact that palivizumab prevents only about $50 \%$ of RSV-related hospital admissions. ${ }^{5,14}$

Our findings support analyses indicating that eliminating 1 of 5 monthly doses and initiating a reduced-dose schedule based on the average season onset would not result in a substantial decline in protection. ${ }^{4}$ Our data are also in keeping with the most recent guidelines of the Academy of Pediatrics and the Canadian Paediatric Society suggesting that fewer than 5 palivizumab doses may provide sufficient seasonal protection in infants discharged after the start of the RSV season., ${ }^{2,3}$ To the best of our knowledge, $\mathrm{BC}$ is the only jurisdiction that has universally adopted this abbreviated schedule.

Critical congenital heart disease is newly diagnosed in nearly 1500 children each year in Canada. ${ }^{16}$ For some of these families, the logistics of travel to clinics during the winter can be considerable, particularly in the geographical context of BC. In our cohort, 42 palivizumab courses were not received (12 cases) or were partially received (30 cases). Of the 42 courses, 31 were missed essentially because parents declined or were not able to attend RSV Immunoprophylaxis Program clinics. Reducing the need for unnecessary treatments can help families focus on other visits that may truly benefit their child. Other benefits include reduction of the direct drug cost and other savings conferred by an abbreviated palivizumab schedule.

The decision of the BC RSV Immunoprophylaxis Program to administer an abbreviated course of palivizumab was based largely on pharmacokinetic data. However, the pharmacokinetics of palivizumab may not be the only reason why fewer doses appear to be effective. Indeed, we previously suggested that neutralizing RSV antibodies persist in children beyond the interval that would be expected on the sole basis of drug pharmacokinetics. ${ }^{17}$ Therefore, it appears that palivizumab serum levels are not the only factor conferring protection against RSV toward the end of the season and that antibodies naturally acquired from clinically unapparent exposure to the virus at the peak of the season may provide substantial additional protection toward the end of the season. ${ }^{17}$

\section{Strengths and limitations}

Major strengths of our study are its population-based nature and high follow-up rates (> 99\%) under the centralized RSV Immunoprophylaxis Program, the cross-referencing of our program data with a provincial hospital discharge database, intention-to-treat analysis and inclusion of a 6-month (Nov. 1 to Apr. 30) fixed-date observation, which spans before and after the drug administration periods. We achieved further robustness by reporting data over multiple years, which facilitates generalization across seasons; this is useful considering the biennial pattern of RSV epidemics. ${ }^{15}$ In terms of limitations, our data from a single province mandate confirmation in other jurisdictions. Second, in the absence of a direct comparison group, it is impossible to ascertain the number of RSV-related hospital admissions that might have been prevented if a standard 5-dose palivizumab regimen had been used. Regarding this latter point, with only 1 admission that occurred outside the palivizumab dosing period, it is extremely unlikely that additional palivizumab doses would have statistically significantly changed our program's outcomes. Moreover, our outcomes are comparable to those of a randomized controlled trial in children with congenital heart disease. ${ }^{5}$ The Canadian Registry of Palivizumab reported 260 hospital admissions, including 35 cases in which RSV testing gave a positive result, among 1909 children over a 10-year period (2005-2015) elsewhere in Canada, where 5 doses are offered; ${ }^{18}$ however, RSV testing was not done in 31 (15\%) of 211 admissions. Moreover, the Canadian Registry of Palivizumab is not a truly population-based registry. We combined RSV-confirmed and RSV-undetermined admissions, which may have overestimated rates of RSVrelated admission in our study. When we consider these factors, rates of RSV-related admission among children with 
congenital heart diseases in BC do not appear different from those in the rest of Canada or in other populations (Appendix 1, available at www.cmajopen.ca/content/7/1/E88/ suppl/DC1). Finally, our experience may not apply to areas where RSV infection epidemics occur in a nonseasonal manner, such as Florida and Alaska, and in other tropical or subtropical areas. ${ }^{19}$

\section{Conclusion}

Our experience in BC shows the protection achieved by a fixed-date program with an abbreviated 4-dose schedule in children with congenital heart disease. These data have a strong theoretical underpinning and warrant consideration in jurisdictions with seasonal RSV infection. It is important to point out that RSV was responsible for only $19 \%$ of admissions for LRTI in our cohort. Therefore, it is critical to continue to emphasize other preventive measures, including family education toward proper hand hygiene, breast-feeding and limiting infectious exposures in children at high risk.

\section{References}

1. Shi T, McAllister DA, O'Brien KL, et al.; RSV Global Epidemiology Network. Global, regional, and national disease burden estimates of acute lower respiratory infections due to respiratory syncytial virus in young children in 2015: a systematic review and modelling study. Lancet 2017;390:946-58.

2. American Academy of Pediatrics Committee on Infectious Diseases; American Academy of Pediatrics Bronchiolitis Guidelines Committee. Updated guidance for palivizumab prophylaxis among infants and young children at increased risk of hospitalization for respiratory syncytial virus infection [published erratum in Pediatrics 2014;134:1221]. Pediatrics 2014;134:415-20.

3. Robinson JL, Le Saux N; Infectious Diseases and Immunization Committee, Canadian Paediatric Society. Preventing hospitalizations for respiratory syncytial virus infection. Paediatr Child Health 2015;20:321-33.

4. Weinberger DM, Warren JL, Steiner CA, et al. Reduced-dose schedule of prophylaxis based on local data provides near-optimal protection against respiratory syncytial virus. Clin Infect Dis 2015;61:506-14.

5. Feltes TF, Cabalka AK, Meissner HC, et al.; Cardiac Synagis Study Group. Palivizumab prophylaxis reduces hospitalization due to respiratory syncytial virus in young children with hemodynamically significant congenital heart disease. $\mathcal{F}$ Pediatr 2003;143:532-40.

6. Subramanian KN, Weisman LE, Rhodes T, et al. Safety, tolerance and pharmacokinetics of a humanized monoclonal antibody to respiratory syncytial virus in premature infants and infants with bronchopulmonary dysplasia. MEDI-493 Study Group. Pediatr Infect Dis 7 1998;17:110-5.

7. Gutfraind A, Galvani AP, Meyers LA. Efficacy and optimization of palivizumab injection regimens against respiratory syncytial virus infection. $7 A M A$ Pediatr 2015;169:341-8.

8. Panozzo CA, Hampp C. Editorial commentary: Can a reduced-dose prophylaxis schedule provide adequate coverage against respiratory syncytial virus infection? Clin Infect Dis 2015;61:515-6.

9. Zaaijer HL, Vandenbroucke-Grauls CM, Franssen EJ. Optimum dosage regimen of palivizumab? Ther Drug Monit 2002;24:444-5.

10. Solimano A, Kwan E. Revisiting palivizumab (PVZ) dosing recommendations based on published pharmacokinetics data [abstract]. Pediatric Academic Societies annual meeting; 2012 Apr. 28-May 1; Boston.

11. Lavoie PM, Solimano A, Taylor R, et al. Outcomes of respiratory syncytial virus immunoprophylaxis in infants using an abbreviated dosing regimen of palivizumab. 7AMA Pediatr 2016;170:174-6.
12. Resch B, Brunner K, Rödl S. Characteristics of severe RSV infection needing intensive care. Eur F Pediatr 2018;177:1407-8.

13. Marr N, Wang TI, Kam SH, et al. Attenuation of respiratory syncytial virusinduced and RIG-I-dependent type I IFN responses in human neonates and very young children. 7 Immunol 2014;192:948-57.

14. Palivizumab, a humanized respiratory syncytial virus monoclonal antibody, reduces hospitalization from respiratory syncytial virus infection in high-risk infants. The IMpact-RSV Study Group. Pediatrics 1998;102:531-7.

15. Lyon JL, Stoddard G, Ferguson D, et al. An every other year cyclic epidemic of infants hospitalized with respiratory syncytial virus. Pediatrics 1996; 97:152-3.

16. Wong KK, Fournier A, Fruitman DS, et al. Canadian Cardiovascular Society/ Canadian Pediatric Cardiology Association position statement on pulse oximetry screening in newborns to enhance detection of critical congenital heart disease. Can 7 Cardiol 2017;33:199-208.

17. Claydon J, Sur A, Callejas A, et al. Respiratory syncytial virus-neutralizing serum antibody titers in infants following palivizumab prophylaxis with an abbreviated dosing regimen. PLoS One 2017;12:e176152.

18. Li A, Wang DY, Lanctôt KL, et al.; CARESS Investigators. Comparing firstand second-year palivizumab prophylaxis in patients with hemodynamically significant congenital heart disease in the CARESS Database (2005-2015). Pediatr Infect Dis 7 2017;36:445-50.

19. Obando-Pacheco P, Justicia-Grande AJ, Rivero-Calle I, et al. Respiratory syncytial virus seasonality: a global overview. 7 Infect Dis 2018;217:1356-64.

Affiliations: Children's \& Women's Health Centre of British Columbia (Claydon, Popescu, Christopherson, Human, Solimano, Lavoie); Divisions of Neonatology (Popescu, Shaiba, Solimano, Lavoie) and Cardiology (Human), Department of Pediatrics, University of British Columbia; British Columbia Children's Hospital Research Institute (Popescu, Human, Lavoie), Vancouver, BC; Victoria General Hospital (Taylor), Victoria, BC; University of Toronto (Shaiba), Toronto, Ont.

Contributors: Jennifer Claydon, Alfonso Solimano and Pascal Lavoie conceived the study, and Alfonso Solimano designed the study. Jennifer Claydon, Lana Shaiba, Cheryl Christopherson and Richard Taylor collected the data, and Jennifer Claydon, Constantin Popescu, Lana Shaiba, Derek Human and Pascal Lavoie analyzed the data. Jennifer Claydon, Alfonso Solimano and Pascal Lavoie interpreted the data. Pascal Lavoie and Alfonso Solimano drafted the manuscript. All of the authors critically revised the manuscript for important intellectual content, gave final approval of the version to be published and agreed to be accountable for all aspects of the work.

Funding: This study was partly funded by the RSV Immunoprophylaxis Program, the British Columbia Lung Association and the Canadian Institutes of Health Research (grant MOP-123478 to Pascal Lavoie). Jennifer Claydon was funded by the British Columbia Children's Hospital. Pascal Lavoie is supported by Investigator Awards from the British Columbia Children's Hospital and the Michael Smith Foundation for Health Research.

Acknowledgements: The authors thank all the practitioners in the British Columbia RSV Immunoprophylaxis Program who made this study possible, George Scott MacRae, consultant, Provincial Health Services Authority Performance Measurement and Reporting, who provided the Discharge Abstract Database cross-referencing data, and Laurence Bayzand, director of Intercampus Operations, British Columbia Children's Hospital and British Columbia Women's Hospital and Health Centre, for his direction, vision and support of the RSV Immunoprophylaxis Program.

Supplemental information: For reviewer comments and the original submission of this manuscript, please see www.cmajopen.ca/content/7/1/ E88/suppl/DC1. 